\title{
Review: migrants are at increased risk of developing schizophrenia
}

Cantor-Graae E, Selten JP. Schizophrenia and migration: A meta-analysis and review. Am J Psychiatry 2005; 162:12-24.

\section{Is migration a risk factor for schizophrenia?}

\section{METHODS}

$-\sqrt{4}$

Design: Systematic review with meta-analysis

Data sources: MEDLINE (search date 2003), database of schizophrenia incidence studies provided by Dr John McGrath (Brisbane, Australia), bibliographies from identified articles were cross referenced.

(1) Study selection and analysis: Studies were included: if schizophrenia incidence rates were reported for one or more migrant groups in a particular area, or data were provided for these calculations; if corrections for age differences between the immigrant group and the reference group were included, or data were provided for these calculations; and if the study was published in an English language, peer reviewed journal. If studies within the same region or time frame were found, only one was included unless the overlap between the studies was small.

Outcomes: Incidence of schizophrenia

\section{MAIN RESULTS}

Eighteen studies met inclusion criteria. The risk of developing schizophrenia was greater in first and second generation migrants compared with the native population (see table).

\section{CONCLUSIONS}

First or second generation migrants are at increased risk of developing schizophrenia compared with the native population. Further investigation is needed into environmental factors that may contribute to this risk.

Table Risk of schizophrenia in migrant populations

\begin{tabular}{ll}
\hline & RR (95\% Cl) \\
\hline First generation migrants & $2.7(2.3$ to 3.2$)$ \\
Second generation migrants & $4.5(1.5$ to 13.1$)$ \\
First and second generation migrants & $2.9(2.5$ to 3.4$)$ \\
\hline
\end{tabular}

For correspondence: Dr Cantor-Graae Department of Community Medicine Lund University, University Hospital UMAS, Malmö, Sweden S-205 02 elizabeth.cantor-graae@smi.mas.lu.se

Sources of funding: Stanley Medical Research Institute.

\section{NOTES}

There was significant heterogeneity between studies for first generation migrants $(\mathrm{p}<0.05)$ and for first and second generation migrants combined $(\mathrm{p}<0.04)$, which reduces the reliability of the results. People at risk of schizophrenia may be more likely to migrate and diagnostic ascertainment of schizophrenia may differ between countries, confounding the studies.

\section{Commentary}

dentifying risk factors for schizophrenia is an area of active research. Many studies have implicated migration as a likely risk factor, although the causal mechanisms remain unclear. Cantor-Graae and Selten provide a meta-analysis of 18 population based studies (1977-2003) from which relative risks were calculated. Their results reveal that both first and second generation migrants have an increased risk for the diagnosis of schizophrenia. When considering 50 effect sizes combining both groups, the mean relative risk is 2.9. Additionally, effect sizes are greater for migrants from developing countries and from areas where the majority of the population is black.

The authors discuss the potential mediators of the association between migration and schizophrenia risk, arguing that selective migration is an unlikely explanation. They conclude that the probable underlying mechanism is one of perceived social inequality and "social defeat". Thus, an ethnic disadvantage leading to psychosocial stress (which impacts neural systems) in genetically predisposed individuals may account for the increased risks associated with migration and especially with migration among those from developing countries and from countries where the population is predominantly black. This exposition is consistent with the stress diathesis model. ${ }^{1}{ }^{2}$

Further population based research is needed, given the limitations of the studies to date, including the dearth of research from countries aside from the UK, the Netherlands, Scandinavia, and Australia, and the fact that few studies have examined all migrant groups in a given country. Compared with the effect sizes for other putative risk factors, migration appears to be an important population level risk factor for schizophrenia. This meta-analysis is particularly notable because it provides estimates of the effect sizes and seeks to further elucidate the aetiologic mechanisms, particularly in terms of psychosocial adversity. Future selective preventive interventions, ${ }^{3}$ targeting a subgroup of individuals at elevated risk, may benefit from focusing on individuals with psychosocial stress related to migration.

Michael T Compton, MD, MPH Emory University School of Medicine, Department of Psychiatry and Behavioral Sciences, Atlanta, Georgia, USA

1 Walker EF, Diforio D. Schizophrenia: a neural diathesis-stress model. Psychol Rev 1997; 104:667-85.

2 Corcoran $C$, Walker $E$, Huot $R$, et al. The stress cascade in schizophrenia: etiology and onset. Schizophr Bull 2003;29:671-92.

3 Mrazek PJ, Haggerty RJ, eds. Reducing risks for mental disorders: frontiers for preventive intervention research. Washington DC: Institute of Medicine, National Academy Press, 1994. 